\title{
THE COMPUTATION OF STATIONARY DISTRIBUTIONS OF MARKOV CHAINS THROUGH PERTURBATIONS ${ }^{1}$
}

\author{
JEFFREY J. HUNTER ${ }^{2}$ \\ Department of Mathematics and Statistics, Massey University \\ Palmerston North, New Zealand
}

\begin{abstract}
An algorithmic procedure for the determination of the stationary distribution of a finite, $m$-state, irreducible Markov chain, that does not require the use of methods for solving systems of linear equations, is presented. The technique is based upon a succession of $m$, rank one, perturbations of the trivial doubly stochastic matrix whose known steady state vector is updated at each stage to yield the required stationary probability vector.
\end{abstract}

Key words: Finite state Markov chains, perturbations, stationary probability vector.

AMS(MOS) subject classification: $\quad 60 \mathrm{~J} 10$.

\section{INTRODUCTION}

In recent years, widespread attention has been given to the computation of stationary distributions of Markov chains. A variety of methods have been suggested and implemented. Before considering an alternative way for finding such distributions, it is of interest to give a brief survey of the techniques that have been employed.

Paige, Styan and Wachter (1975) presented a comprehensive survey of eight different algorithms involving a variety of procedures including the use of generalized inverses, rank reduction, least squares and power methods. Their recommendation was a direct method that involved transforming the singular set of stationary equations into a non-singular system using a rank one modification followed by Gaussian elimination with row pivoting. A further study by

\footnotetext{
${ }^{1}$ Received: January 1990, Revised: September 1990.
}

${ }^{2}$ This research was supported in part by the Air Force Office of Scientific Research under contract F49620 85 C 0144 through the Center for Stochastic Processes, Department of Statistics, University of North Carolina, Chapel Hill, North Carolina, 27514, USA. 
Harrod and Plemmons (1984) provided another direct approach based upon the LU factorization using Gaussian elimination without pivoting.

Iterative techniques and approximation methods have been surveyed by Koury, McAllister and Stewart (1984). When the transition matrix is large and exhibits a nearly completely decomposable structure it is shown that a method of "aggregation" can be combined with point and block iterative techniques to produce methods which converge rapidly to the stationary probability vector.

Sheskin (1985) presented a partitioning algorithm that used a matrix reduction routine that partitions the transition matrix to create a sequence of smaller order transition matrices followed by a vector enlargement routine that enables the components of the steady state vector to be determined sequentially. A related procedure was developed by Grassmann, Taksar and Heyman (1985) using the theory of regenerative processes. They derived relationships between the steady state probabilities which are then used to develop a numerical algorithm to find these probabilities. Both of these latter two techniques appear to be, in effect, modifications of Gaussian elimination.

More recently, Meyer (1987), has utilized the concept of "stochastic complementation" whereby an irreducible chain is uncoupled into smaller irreducible chains whose stationary distributions can be coupled back together to produce the stationary distribution of the original chain.

In this paper, an entirely new approach involving the analysis of perturbed Markov chains is considered. In Hunter (1986), techniques for updating the stationary distribution of a finite irreducible Markov chain, following a rank one perturbation of its transition matrix, were presented. In this current paper, these techniques are utilized, to construct a general procedure for determining the stationary distribution of any finite irreducible Markov chain. A significant feature of the proposed algorithm is that at no stage does a system of linear equations have to be solved and consequently there is no reliance upon computer subroutines for matrix inversion or, the more generally accepted method of solution, Gaussian elimination with or without pivoting.

The basic idea is very simple. Suppose the steady state probability vector $\pi^{\prime}$ of an $m$-state irreducible Markov chain with given transition matrix $P$ is required. Let $P_{0}$ be the transition matrix of another irreducible, $m$-state, Markov chain with known stationary probability vector $\underset{\sim}{\sim} 0^{\prime}$. By replacing, successively, the elements of each row of $P_{0}$ with the corresponding row elements as specified by $P$ and recomputing the stationary probability vector of the resultant perturbed transition matrix, the vector $\underset{\sim}{\pi}{ }_{0}^{\prime}$ can be transformed, in $m$ stages, to ${\underset{\sim}{\pi}}^{\prime}$, by a series of $m$ updates. 
As the irreducibility of a Markov chain is governed by the location of the positive entries in its transition matrix, to ensure the irreducibility of each perturbed Markov chain it is sufficient to commence with $P_{0}$ containing positive elements placed at least in the same position as those in $P$.

Consider starting with the trivial doubly stochastic matrix $P_{0}$ with each element having the value $1 / m$, so that $P_{0}=\underset{\sim}{e} e^{\prime} / m$, where ${\underset{\sim}{e}}^{\prime}=(1,1, \ldots, 1)$ is a vector of ones. As can be easily shown, $\underset{\sim}{\sim}{ }_{0}^{\prime}=e^{\prime} / m$.

For $i=1,2, \ldots, m$, let $\underset{\sim}{e}$ be the $i^{\text {th }}$ elementary (column) vector with a one in the $i^{\text {th }}$ position and zeros elsewhere. Let $\underset{\sim}{p}{ }_{i}^{\prime}={\underset{\sim}{e}}_{i}^{\prime} P$ be the $i^{\text {th }}$ row of $P$ and let

$$
P_{i}=P_{i-1}+e_{i} \stackrel{b}{\sim_{i}^{\prime}}
$$

where

$$
\stackrel{b}{\sim}_{i}^{\prime}=\underset{\sim}{p}{ }_{i}^{\prime}-e^{\prime} / m
$$

Let $\underset{i}{\sim}$ be the stationary probability vector associated with the Markov chain with transition matrix $P_{i}$, and, since $P_{m}=_{i=1}^{m} \underset{\sim}{e} \underset{\sim}{p}{ }_{i}^{\prime}=P, \underset{\sim}{\prime}{ }_{m}^{\prime}$ is in fact the required vector $\pi^{\prime}$.

\section{GENERAL THEORY}

The construction of the algorithm is based upon the following results.

Theorem 2.1. Let $P_{i}$ be the transition matrix of a finite irreducible Markov chain with stationary probability vector $\pi_{i}^{\prime}$.

(a) $\quad I-P_{i}+\underset{\sim}{i} \underset{i}{\sim}{ }_{i}^{\prime}$ is non-singular if and only if ${\underset{\sim}{u}}_{i}^{\prime} \underset{\sim}{e} \neq 0$ and $\pi_{i}^{\prime}{ }_{i}^{t} \neq 0$.

(b) Under the conditions of $(a)$,

$$
\stackrel{\pi}{\sim}_{i}^{\prime}=\alpha_{i}^{\prime} /{\underset{\sim}{\alpha}}_{i}^{\prime} \underset{\sim}{e}
$$

where

$$
{\underset{\sim}{i}}_{i}^{\prime}={\underset{\sim}{u}}_{i}^{\prime}\left[I-P_{i}+\underset{\sim}{i} \underset{i}{\sim_{i}^{\prime}}\right]^{-1}
$$

Proof. For (a) see Theorem 3.3 in Hunter (1982) and for (b) see Corollary 4.1.2 in Hunter (1982).

Theorem 2.2. If $X$ is non-singular and $\underset{\sim}{b} X^{-1} \underset{\sim}{\sim} \neq-1$, then

$$
\left(X+\underset{\sim}{a} \underline{\sim}^{\prime}\right)^{-1}=X^{-1}-\frac{X^{-1} \underset{\sim}{a} \underset{\sim}{b}{ }^{\prime} X^{-1}}{1+\underline{\sim}^{\prime} X^{-1} \underset{\sim}{a}} \text {. }
$$


Proof. This is the Sherman-Morrison formula. See Golub and Van Loan (1983), p. 3.

Suppose that, following the $i^{t h}$ perturbation, the stationary probability vector $\pi_{i}^{\prime}$ has been found for the Markov chain with transition matrix $P_{\mathfrak{i}}$, as given by (1.1), by using the procedure described by Theorem $2.1(b)$ for suitable choices of $\underset{\sim}{t} i$ and $\underset{\sim}{u} i$.

In Hunter (1986) it was shown that it is possible to find an expression for $\pi^{\prime}{ }_{i+1}$, associated with $P_{i+1}$, using the same procedure outlined in Theorem $2.1(b)$, by choosing the $\underset{\sim}{t} i+1$ and $\underset{\sim}{u} i+1$ in such a way that $\left[I-P_{i+1}+\underset{\sim}{i} i+1{\underset{u}{\sim}}^{\prime}{ }_{i+1}\right]^{-1}$ can be determined from the earlier deduced expression $\left[I-P_{i}+\underset{\sim}{i} \underset{\sim}{\sim}{ }_{i}^{\prime}\right]^{-1}$, without performing an additional matrix inversion.

For the particular situation under consideration, for $i=0,1, \ldots, m-1, \underset{\sim}{\underset{\sim}{t} i+1}{ }_{i+e_{i+1}}$ and $\stackrel{u}{\sim}_{i+1}={\underset{u}{\sim}}_{i}+\underline{b}_{i+1}$, where ${\underset{b}{\sim}}_{i}$ is given by (1.2), then, from (1.1),

$$
\begin{aligned}
& I-P_{i+1}+\underset{\sim}{\sim} i+1 \underline{u}^{\prime} i+1=I-P_{i}+\underset{\sim}{i} i+1 \stackrel{u}{\sim}_{i}^{\prime}, \\
& =I-P_{i}+\underline{t}_{i}{\underset{\sim}{u}}_{i}^{\prime}+\left({\underset{t}{i}}_{i+1}-\underline{\sim}_{i}\right) \underline{u}_{i}^{\prime}
\end{aligned}
$$

Now if $\left.\left[I-P_{i}+\underset{\sim}{\sim} \underset{\sim}{\sim}\right]_{i}^{\prime}\right]^{-1}$ exists, from the proof of Theorem 3.3 in Hunter (1982),

$$
\left.\stackrel{u}{\sim}_{i}^{\prime}\left[I-P_{i}+\underset{\sim}{i}{ }_{i}^{u}\right]_{i}^{\prime}\right]^{-1}=\frac{\pi_{i}^{\prime}}{{\underset{\sim}{\sim}}_{i}^{\prime} \underset{\sim}{t}}
$$

Thus, using (2.3), (2.4) and (2.5), if $A_{i} \equiv\left[I-P_{i}+\underset{\sim}{i} \underset{\sim}{\sim} \underset{i}{\prime}\right]^{-1}$ exists,

$$
A_{i+1}=A_{i}\left[I+\left({\underset{\sim}{i}}_{i}-\underline{\sim}_{i+1}\right) \frac{\pi_{i}^{\prime}}{\pi_{i}^{\prime} \stackrel{t}{i+1}_{i+1}}\right]
$$

Equation (2.6) is ideally suited for recursive operations once an initial inverse $A_{0}=\left[I-P_{0}-\underline{\sim} 0 \stackrel{u}{\sim}_{0}^{\prime}\right]^{-1}$ has been determined. However, because of the form of $P_{0}$ that has been selected, if $\underset{\sim}{t} 0=e$ and $\underset{\sim}{u}{ }_{0}^{\prime}=e^{\prime} / m$, no matrix inverse has to be computed since, in this instance,

$$
I-P_{0}+\underset{\sim}{\sim} 0 \underset{\sim}{u}{ }_{0}^{\prime}=I-\underset{\sim}{e} \stackrel{\sim}{e}^{\prime} / m+\underset{\sim}{e} \stackrel{\sim}{\sim}^{\prime} / m=I
$$

Furthermore, using (2.2),

$$
\alpha_{0}^{\prime}=\underline{u}_{0}^{\prime}=e^{\prime} / m
$$

and, from (2.1),

$$
\pi_{0}^{\prime}=e^{\prime} / m
$$


The basic algorithmic procedure now follows:

$$
\text { Let } \underset{\sim}{t} 0=\underset{\sim}{e}, \underline{\sim}_{0}^{\prime}=e^{\prime} / m, A_{0}=I, \pi_{0}^{\prime}=e^{\prime} / m
$$

For $i=1,2, \ldots, m$, let $\underset{\sim}{t} i=\underset{\sim}{e}$, and ${\underset{\sim}{u}}_{i}^{\prime}={\underset{\sim}{u}}_{i-1}^{\prime}+{\underset{\sim}{p}}_{i}^{\prime}-\underline{\sim}^{\prime} / m$.

Compute $\quad A_{i}=A_{i-1}\left[I+\left(\stackrel{t}{\sim}_{i-1}-\underline{t}_{i}\right) \pi^{\prime}{ }_{i-1} / \stackrel{\pi}{\sim}^{\prime} i-1 \stackrel{t}{\sim} i\right]$.

Compute

$$
{\underset{\sim}{\alpha}}_{i}^{\prime}={\underset{\sim}{u}}_{i}^{\prime} A_{i}
$$

Compute

$$
\tilde{\pi}_{i}^{\prime}={\underset{\sim}{\alpha}}_{i}^{\prime} / \alpha_{i}^{\prime} \underset{\sim}{e}
$$

Then $\pi^{\prime}=\pi{ }_{m}^{\prime}$ is the stationary probability vector of the Markov chain with transition matrix $P=\left[p_{i j}\right]$.

Since the elements of any stationary probability vector are always positive, ${\underset{\pi}{\sim}}_{i}^{\prime} \underset{\sim}{t} i$ and $\pi^{\prime}{ }_{i-1} \stackrel{t}{\sim}_{i}$ are both positive. Further, by induction, for $i=0,1, \ldots, m$,

$$
\stackrel{u}{\sim}_{i}^{\prime} \underset{\sim}{e}=1
$$

so that the conditions of Theorem 2.1 and 2.2 are satisfied.

\section{REFINEMENTS TO THE ALGORITHM}

Although the procedure suggested in Section 2 will lead to the required stationary probability vector there are some modifications that, if employed, will lead to a more efficient procedure.

\subsection{MODIFICATION TO THE $\underset{\sim}{\pi}$ COMPUTATION}

The ultimate aim of the algorithm is to determine $\pi^{\prime}=\underset{\sim}{\pi}$. Unless the stationary distributions of the intermediate perturbed Markov chains are required, some simplification can be effected by observing that (2.7) requires $\pi_{\sim}{ }_{i-1}$ through its scaled version $\pi^{\prime}{ }_{i-1} / \pi^{\prime}{ }_{i-1} \underset{\sim}{\sim}{ }_{i}$. The scaling suggested by (2.9) is not required until the final step when $i=m$.

Thus, for $i=0,1, \ldots, m-1$, let

$$
\stackrel{v}{\sim}_{i}^{\prime} \equiv \pi_{i}^{\prime} / \underline{\pi}_{i}^{\prime} \underset{\sim}{i} i+1
$$

Then, $\underset{\sim}{v} \underset{0}{\prime}=e^{\prime}$ and for $i=1,2, \ldots, m-1$

$$
\stackrel{v}{\sim}_{i}^{\prime}={\underset{\sim}{u}}_{i}^{\prime} A_{i} / \underline{u}_{i}^{\prime} A_{i} \underset{\sim}{e} i+1
$$


If $\pi_{i}^{\prime}$ is required then it can be recovered simply. For $i=0,1, \ldots, m-1$

$$
\stackrel{\pi}{\sim}_{i}^{\prime}={\underset{\sim}{v}}_{i}^{\prime} / \underline{\sim}_{i}^{\prime} \underset{\sim}{e}
$$

At the final step, compute $\underset{\sim}{\prime} m$ using (2.8) and (2.9).

\subsection{MODIFICATION TO THE $\boldsymbol{A}_{\boldsymbol{i}}$ COMPUTATION}

With the notation introduced in Section 3.1 , it is easily seen that the early terms in the $\left\{A_{i}\right\}$ sequence are given, after simplification, as

$$
\begin{aligned}
& A_{0}=I \\
& A_{1}=A_{0}+\left(\underset{\sim}{e}-\underline{\sim}_{1}\right) \underset{\sim}{v}{ }_{0}^{\prime} \\
& A_{2}=A_{1}+\left(e_{1}-\sim_{2}\right){ }_{1}^{\prime}, \\
& A_{3}=A_{2}+\left[\left(1-v_{13}\right) \underset{\sim}{\sim}{ }_{1}+v_{13} \underset{\sim}{\sim}{ }_{2}-\underset{\sim}{e}\right] \underset{\sim}{\sim}{ }_{2}^{\prime}, \\
& A_{4}=A_{3}+\left[\left\{1-v_{14}-\left(1-v_{13}\right) v_{24}\right\} \stackrel{e}{\sim}_{1}\right. \\
& \left.+\left(v_{14}-v_{13} v_{24}\right) \underset{\sim}{\stackrel{e}{2}}+v_{24} \underset{\sim}{\stackrel{e}{\sim}}{ }_{3}-\underset{\sim}{\sim}\right]_{4} \underset{\sim}{v_{3}^{\prime}},
\end{aligned}
$$

where $v_{i j} \equiv{\underset{\sim}{v}}_{i}^{\prime} \underset{j}{j}$, so that $v_{i, i+1}=1$ for $i=1,2, \ldots, m-1$

The above results provide motivation for the following theorem.

Theorem 3.1. For $n=0,1, \ldots, m-1$,

$$
A_{n+1}=A_{n}+B_{n}
$$

where

$$
B_{n}=A_{n}\left({\underset{\sim}{\sim}}_{n}-e_{n+1}\right) \underset{\sim}{\sim}{ }_{n}^{\prime}{\underset{\sim}{\sim}}_{n}{\underset{\sim}{v}}_{n}^{\prime}
$$

with $\underset{\sim}{e} 0 \equiv \underset{\sim}{e}$, so that $\underset{\sim}{b} 0=\underset{\sim}{e}-{\underset{\sim}{e}}_{1}$ and for $n=1,2, \ldots, m-1$

$$
\stackrel{b}{\sim}_{n}=b_{1 n} \stackrel{e}{\sim}_{1}+\ldots+b_{n n} \stackrel{e}{\sim}_{n}-e_{n+1}
$$

Proof. The theorem is obviously true by inspection, from (3.4) to (3.7), for $n=0,1,2,3$. Assume that (3.9) and (3.10) hold for $n=0,1, \ldots . k$ so that 


$$
\begin{gathered}
A_{k+1}=A_{k}+B_{k}=A_{k-1}+B_{k-1}+B_{k} \\
=\ldots=I+\sum_{n=}^{k} B_{n} .
\end{gathered}
$$

Hence

$$
\begin{gathered}
B_{k+1}=A_{k+1}(\underbrace{}_{k+1}-\mathcal{L}_{k+2}) \mathcal{v}^{\prime}{ }_{k+1}, \\
=\left(I+B_{0}+\sum_{n=1}^{k} B_{n}\right)(e_{k+1}-\underbrace{}_{k+2}) \sim^{\prime}{ }_{k+1},
\end{gathered}
$$

implying that

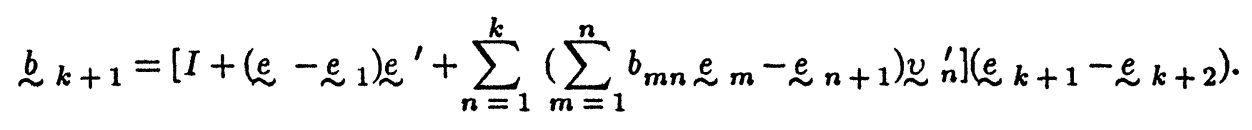

Since $\stackrel{e}{\sim}^{\prime}(\underset{\sim}{e} k+1-\underset{\sim}{k} k+2)=0$

$$
\begin{aligned}
& \stackrel{b}{\sim}_{k+1}={\underset{\sim}{e}}_{k+1}-\underset{\sim}{\sim} k+2+\sum_{n=1}^{k}\left(\sum_{m=1}^{n} b_{m n} \stackrel{e}{\sim}_{m}-\stackrel{e}{\sim}_{n+1}\right)\left(v_{n, k+1}-v_{n, k+2}\right), \\
& =\underbrace{}_{k+1}-\underset{\sim}{e} k+2+\sum_{m=1}^{k}\left\{\sum_{n=m}^{k} b_{m n}\left(v_{n, k+1}-v_{n, k+2}\right)\right\}_{\sim} \underset{m}{ } \\
& -\sum_{m=2}^{k+1}\left(v_{m-1, k+1}-v_{m-1, k+2)} e_{m}\right.
\end{aligned}
$$

showing that $\underset{\sim}{b} k+1$ is linear combination of $\underset{\sim}{e}{ }_{1}, \ldots, \underset{\sim}{e} k+2$ with $\underset{\sim}{\sim} k+2$ having coefficient -1 . Thus (3.10) is true for $n=k+1$ and the theorem follows by induction.

Observe that

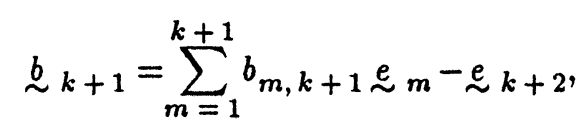

where

$$
\begin{gathered}
b_{1, k+1}=\sum_{n=1}^{k} b_{1, n}\left(v_{n, k+1}-v_{n, k+2}\right), \\
b_{k+1, k+1}=v_{k, k+2},\left(\text { since } v_{k, k+1}=1\right),
\end{gathered}
$$

and for $m=2, \ldots, k$, 


$$
b_{m, k+1}=\sum_{n=m-1}^{k} b_{m, n}\left(v_{n, k+1}-v_{n, k+1}\right) .
$$

Note that

$$
\begin{aligned}
& B_{n}=\underline{b}_{n} \underline{\sim}_{n}^{\prime}=\left[\begin{array}{c}
b_{1 n} \\
b_{2 n} \\
\cdot \\
\cdot \\
b_{n n} \\
-1 \\
0 \\
\cdot \\
0
\end{array}\right] \quad\left[v_{n 1}, v_{n 2}, \ldots, v_{n m}\right]
\end{aligned}
$$

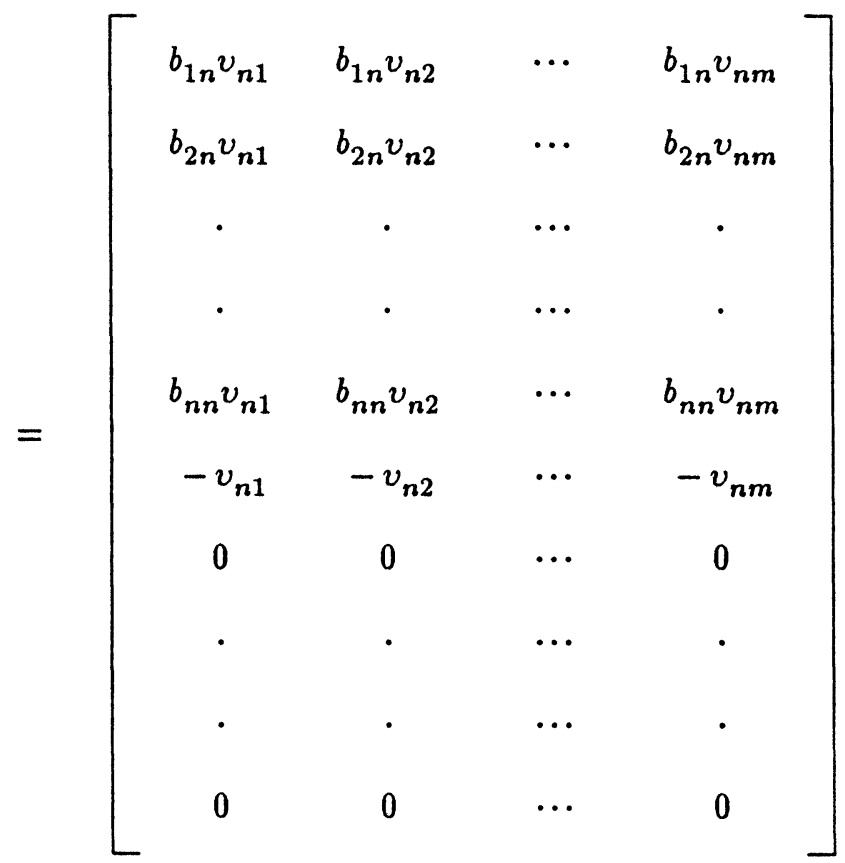

Thus, in the matrix $B_{n}$, all the entries in rows number $n+2, \ldots, m$ are zero. Obviously, this has considerable significance in the calculation of the matrices $A_{i}(i=2, \ldots, m)$ as required in the algorithm. 
The updating process, given by $(2.7)$, can be replaced for $i=2, \ldots, m$, by

$$
A_{i}=A_{i-1}+B_{i-1}
$$

where

$$
B_{i-1}-A_{i-1}\left(\underset{\sim}{e} i-1-\stackrel{e}{\sim}_{i}\right) \stackrel{v}{\sim}_{i-1}^{\prime}
$$

is such that only the first $i$ rows of $B_{i-1}$ are computed with the remaining entries set equal to zero.

Furthermore, from (3.16), some of the rows of $B_{n}$ have a special form and do not require computation. In particular, the $(n+1)$ th row is simply $-\underbrace{\prime}_{n}$ while, from (3.14), the $n^{\text {th }}$ row is $v_{n-1, n+1}$ times $\underset{\sim}{\sim}$.

Note also that the $(n+1)$ st column of $B_{n}$ is $\underset{\sim}{b}$ since $v_{n, n+1}=1$.

There are also some other checks that can be applied.

Theorem 3.2. For $i=1,2, \ldots, m$,

$$
\begin{gathered}
A_{i} e_{i}=\underset{\sim}{\sim} \\
\frac{1}{m} e^{\prime} A_{i}=\underset{\sim}{\sim} \\
\stackrel{e}{\sim}^{\prime} B_{i}=\underset{\sim}{0}
\end{gathered}
$$

Proof. Since $A_{i}=\left[I-P_{i}+\underset{\sim}{i} u_{i}^{\prime}\right]^{-1}$, equation (3.17) of Hunter (1982) implies that

$$
A_{i} \underset{\sim}{i}=\frac{e}{\stackrel{u}{\sim}_{i}^{\prime} e}=e
$$

yielding (3.19) with $\underset{\sim}{t}{ }_{i}=\underset{\sim}{i}$.

Equation (3.20) is obviously true when $i=1$ since

$$
\begin{gathered}
\stackrel{e}{ }^{\prime} A_{1}=\stackrel{e}{ }^{\prime}\left[I+(\underset{\sim}{e}-\underset{\sim}{e}) e^{\prime}\right] \\
=\stackrel{e}{\sim}^{\prime}+(m-1) \stackrel{\sim}{\sim}^{\prime} \\
=m e^{\prime}
\end{gathered}
$$

Thus, by induction, if (3.20) is true for $i=1,2, \ldots, k$, from (2.7) and (3.1), 


$$
\begin{aligned}
& \frac{1}{m} e^{\prime} A_{k+1}=\frac{1}{m} \underset{\sim}{e} A_{k}\left[I+\left({\underset{\sim}{e}}_{k}-\underset{\sim}{e} k+1\right) \underset{\sim}{v}\right] \\
& =\underline{\sim}^{\prime}\left[I+\left({\underset{\sim}{e}}_{k}-{\underset{\sim}{e}}_{k+1}\right){\underset{\sim}{v}}_{k}^{\prime}\right] \\
& =\tilde{e}^{\prime}+\left(\tilde{\sim}^{\prime} e{ }_{k}-\sim^{\prime} \stackrel{\sim}{ }_{k+1}\right) \tilde{v}_{k}^{\prime}=e^{\prime} .
\end{aligned}
$$

Thus (3.20) and hence also (3.21) follow.

A consequence of Theorem 3.2 is that the $i^{\text {th }}$ column of $A_{i}$ consists solely of unit elements while the sum of elements of each column of $B_{i}$ is zero.

\subsection{MODIFICATION TO THE $\underset{\underset{i}{i}}{ }$ COMPUTATION}

Although the $\left\{A_{i}\right\}(i=0,1, \ldots, m)$ sequence plays an integral role in the procedure, the matrices $A_{i}$ are required only to obtain the sequence of vectors $\underset{\sim}{\alpha}{ }_{i}^{\prime}={\underset{\sim}{u}}_{i}^{\prime} A_{i}$ and hence the vectors $\tilde{v}_{i}^{\prime}$. Thus is worth examining whether it is possible to dispense with explicit calculation of the $A_{i}$ by deriving the $\left\{\alpha_{i}^{\prime}\right\}(i=1,2, \ldots, m)$ sequence recursively.

Theorem 3.3. For $i=0,1,2, \ldots, m-1$,

$$
\stackrel{\alpha}{\sim}_{i+1}^{\prime}={\underset{\sim}{v}}_{i}^{\prime}-\underline{\sim}^{\prime}+{\underset{\sim}{p}}_{i+1}^{\prime} A_{i+1}
$$

Proof. First observe that, from (2.8),

$$
{\underset{\sim}{\alpha}}_{1}^{\prime}={\underset{\sim}{u}}_{1}^{\prime} A_{1}=\underset{\sim}{p}{ }_{1}^{\prime} A_{1},
$$

so that (3.22) holds for $i=0$ since $\underset{\sim}{v}{ }_{0}^{\prime}=e^{\prime}$.

In general, for $i=1,2, \ldots, m-1$, from $(2.8)$,

$$
\begin{gathered}
\stackrel{\alpha}{\alpha}^{\prime}{ }_{i+1}=\underline{\sim}_{\sim}^{\prime}{ }_{i+1} A_{i+1}, \\
=\left[{\underset{\sim}{\sim}}_{i}^{\prime}-\left(\sim^{\prime} / m\right)+{\underset{\sim}{p}}^{\prime}{ }_{i+1}\right] A_{i+1} .
\end{gathered}
$$

Now

$$
\begin{aligned}
& {\underset{\sim}{u}}_{i}^{\prime} A_{i+1}={\underset{\sim}{u}}_{i}^{\prime}\left[A_{i}+A_{i}(\underset{\sim}{e} i-\underset{\sim}{e} i+1) \underset{\sim}{\sim}{ }_{i}^{\prime}\right] \\
& =\alpha_{i}^{\prime}+{\underset{\sim}{\alpha}}_{i}^{\prime}\left(\underset{\sim}{e}-\stackrel{e}{\sim}_{i+1}\right){\underset{\sim}{v}}_{i}^{\prime}, \\
& ={\underset{\sim}{\sim}}_{i}^{\prime}+\left(\alpha_{i i}-\alpha_{i, i+1}\right) \underset{\sim}{v},
\end{aligned}
$$

where $\alpha_{i j}=\alpha_{i}^{\prime} \underset{\sim}{e}$. But, from (3.19) and (2.10), 


$$
\alpha_{i i}={\underset{u}{\sim}}_{i}^{\prime} A_{i} \underset{\sim}{\stackrel{e}{\sim}}={\underset{\sim}{u}}_{i}^{\prime} \underset{\sim}{e}=1
$$

and, since from (3.2) and (2.8), ${\underset{\sim}{v}}_{i}^{\prime}={\underset{\sim}{\alpha}}_{i}^{\prime} / \alpha_{i, i+1},(3.24)$ becomes

$$
\stackrel{u}{\sim}_{i}^{\prime} A_{i+1}=\underline{\sim}_{i}^{\prime}
$$

Equation (3.22) now follows from (3.23) upon substitution of (3.26) and (3.20).

Theorem 3.3 shows that in updating from $\underset{\sim}{\alpha}{ }_{i}^{\prime}$ to ${\underset{\sim}{\alpha}}_{i+1}^{\prime}$ the term $\underset{\sim}{p}{ }_{i+1} A_{i+1}$ must be computed. The calculations of $\underset{\sim}{\alpha}{ }_{1}^{\prime}, \underset{\sim}{\alpha}{ }_{2}^{\prime}, \ldots,{\underset{\sim}{\alpha}}_{i}^{\prime}$ require, successively, $\underset{\sim}{p}{ }_{1}^{\prime}, \ldots, \underset{\sim}{p}{ }_{i}^{\prime}$ and for ${\underset{\sim}{\alpha}}_{i+1}^{\prime}$ this is the first time ${\underset{\sim}{p}}^{\prime}{ }_{i+1}$, the $(i+1)$ th row of $P$ is involved.

Although, $\underset{\sim}{p}{ }_{i+1} A_{i+1}$ can be expressed in terms of $A_{i}$, as can be seen from the next theorem, very little advantage is gained since such terms are required for each $i=0,1, \ldots, m-1$.

Theorem 3.4. For $i=0,1, \ldots, m-1$,

$$
\underline{\sim}^{\prime}{ }_{i+1} A_{i+1}=\underline{\sim}^{\prime}{ }_{i+1} A_{i}+\underline{\sim}_{i}^{\prime}-\left(\underline{\sim}_{i+1}^{\prime} A_{i} \underset{\sim}{e} i+1\right){\underset{\sim}{v}}_{i}^{\prime}
$$

Proof. For $i=0$,

$$
\begin{aligned}
& \underset{\sim}{p}{ }_{1}^{\prime} A_{1}=\underset{\sim}{p}{ }_{1}^{\prime}\left[I+\left(\underset{\sim}{e}-{\underset{\sim}{e}}_{1}\right) e_{\sim}^{\prime}\right], \\
= & \underset{\sim}{p}{ }_{1}^{\prime}+\left(\underline{\sim}_{1}^{\prime} \underset{\sim}{e}\right) e^{\prime}-\left(\underline{\sim}_{1}^{\prime} \underset{\sim}{e}\right) e^{\prime},
\end{aligned}
$$

and the result follows, since $\underset{\sim}{p} \underset{\sim}{\prime} e=1, \underset{\sim}{v}{ }_{0}^{\prime}={\underset{\sim}{e}}^{\prime}$ and $A_{0}=I$.

In general, for $i=1,2, \ldots, m-1$, from (2.7) and (3.1),

$$
\stackrel{\sim}{\sim}^{\prime}{ }_{i+1} A_{i+1}=\underline{\sim}_{i+1}^{\prime}\left[A_{i}+A_{i}\left({\underset{\sim}{e}}_{i}-\stackrel{e}{\sim}_{i+1}\right){\underset{\sim}{v}}_{i}^{\prime}\right]
$$

Equation (3.27) follows since, from (3.19),

$$
\stackrel{p}{\sim}_{i+1}^{\prime} A_{i} \underset{\sim}{\sim}{ }_{i}=\underline{\sim}^{\prime}{ }_{i+1} \underset{\sim}{e}=1
$$

As a consequence of Theorem 3.3 , it is suggested that (2.8) in the algorithm be replace by (3.22). 


\subsection{MODIFICATION TO THE $\underset{\sim}{\prime}{ }_{m}^{\prime}=\pi^{\prime}$ COMPUTATION}

At the final step of the algorithm $A_{m}$ can be computed and consequently $\underset{\sim}{\pi}{ }_{m}^{\prime}$ derived as $\underset{\sim}{\alpha} / \underset{\sim}{\alpha}{ }_{m}^{\prime} \underset{\sim}{\sim}$ where $\underset{\sim}{\alpha}{ }_{m}^{\prime}={\underset{\sim}{u}}_{m}^{\prime} A_{m}$. However, $A_{m}$ need not be explicitly determined since, from Theorem 3.3 and 3.4,

$$
\stackrel{a}{\sim}{ }_{m}^{\prime}={\underset{\sim}{v}}_{m-1}^{\prime}-\underline{\sim}^{\prime}+\underset{\sim}{p}{ }_{m}^{\prime} A_{m}
$$

where $\underset{\sim}{p}{ }_{m}^{\prime} A_{m}=\underset{\sim}{p}{ }_{m}^{\prime} A_{m-1}+{\underset{\sim}{v}}_{m-1}^{\prime}-\left(\underset{\sim}{p}{ }_{m}^{\prime} A_{m-1} \underset{\sim}{e}\right){\underset{\sim}{v}}_{m-1}^{\prime}$

\section{RECOMMENDED PROCEDURE}

As a consequence of the refinements discussed in Section 3, it is suggested that the algorithm be constructed as follows:

Let $A_{1}=I+(\underset{\sim}{e}-\underset{\sim}{e})^{\prime} \stackrel{e}{\prime}^{\prime}$

2) Let ${\underset{\sim}{\alpha}}_{1}^{\prime}=\underset{\sim}{p}{ }_{1}^{\prime} A_{1}$.

3) For $i=1,2, \ldots, m-2$, compute

(a) $\quad \underline{v}_{i}^{\prime}={\underset{\sim}{\alpha}}_{i}^{\prime} /{\underset{\sim}{\alpha}}_{i}^{\prime} \stackrel{e}{i+1}_{i}$

(b) $\quad B_{i}=A_{i}\left({\underset{\sim}{\sim}}_{i}-\underset{\sim}{e} i+1\right){\underset{\sim}{\sim}}_{i}^{\prime}$ '

(c) $A_{i+1}=A_{i}+B_{i}$,

(d) $\quad \alpha^{\prime}{ }_{i+1}={\underset{v}{v}}_{i}^{\prime}-\underline{\sim}^{\prime}+{\underset{\sim}{p}}^{\prime}{ }_{i+1} A_{i+1}$.

Let ${\underset{\sim}{v}}_{m-1}^{\prime}=\alpha_{m-1}^{\prime}{ }_{m-1}{ }^{\prime}{ }_{m-1}^{e}$.

5) Let $\underset{\sim}{\alpha}{ }_{m}^{\prime}=2{\underset{\sim}{v}}_{m-1}^{\prime}-{\underset{\sim}{e}}^{\prime}+\underset{\sim}{p}{ }_{m}^{\prime} A_{m-1}-\left(\underset{\sim}{p}{ }_{m}^{\prime} A_{m-1} \underset{\sim}{e}\right){\underset{\sim}{v}}_{m-1}^{\prime}$.

6) Let $\pi^{\prime}=\underset{\sim}{\pi} \stackrel{\alpha}{\alpha}{ }_{m}^{\prime} / \alpha_{m}^{\prime} \underset{\sim}{e}$.

The order of the number of arithmetic operations (multiplication and division) required to determine $\pi^{\prime}$ can be estimated as follows. The computation of the $B_{i}$ and the $\pi_{i}^{\prime} A_{i}$ have a dominant effect on the number of operations required. Since $A_{i}\left(\underset{\sim}{e} i_{i}-\underset{\sim}{e} i+1\right)$ is effectively the difference of two columns of $A_{i}$, only $m i$ operations are required to determine $B_{i}$, taking into consideration that $B_{i}$ has only $(i+1)$ non-zero rows, and, as a consequence of $(3.21)$, that the elements of one row can be found from the other rows using the fact that each column sums to zero. On the other hand, for a general transition matrix, $\underset{\sim}{p}{ }_{i}^{\prime} A_{i}$ will require $m^{2}$ operations, although this can be reduced to $m(m-1)$ since the $i$ th element of this row vector, ${\underset{\sim}{p}}_{i}^{\prime} A_{i} \underset{\sim}{e}{ }_{i}={\underset{\sim}{p}}_{i}^{\prime} \underset{\sim}{e}=1$, (by using (3.19)). Since the other calculations required are relatively insignificant in comparison, the total number of operations is of the order of

$$
\sum_{i=1}^{m-1} m i+\sum_{i=1}^{m} m(m-1)=3 m^{2}(m-1) / 2, \text { i.e. of order } 3 m^{3} / 2 \text {. }
$$


To solve for the stationary distribution directly using Gaussian elimination requires of the order fo $4 \mathrm{~m}^{3} / 3$, while to solve directly using a matrix inversion routine requires of the order of $2 \mathrm{~m}^{3}$ operations, (see Isaacson and Keller (1966)).

The procedure is, in effect, finding the stationary distribution of $m$ different irreducible Markov chains and consequently the routine that has been developed offers much more information than other techniques currently available.

Although it has been suggested that the algorithm proceed row by row, there is no necessity to adhere to a strict sequential ordering of the rows. The procedure as outlined by (2.7), (2.8) and (2.9) can easily be adapted to such changes by altering the $\underset{\sim}{t} i$ and ${\underset{\sim}{u}}_{i}^{\prime}$. A consequence of this is that the effect of changing selected transition probabilities upon the stationary distribution can easily be determined. (See also Hunter (1986)).

The procedure also offers the opportunity to utilize the structure of special transition matrices. For example, if the transition matrix of the chain is banded with $p_{i j}=0$ for $j<i-g$ and $j>i+h$, which occurs in some queueing models, the calculation of $\underset{\sim}{p}{ }_{i} A_{i}$ will require at most $(g+h) m$ operations and the algorithm will require on the order of only $\mathrm{m}^{3} / 2+(g+h) \mathrm{m}^{2}$ operations.

\section{STRUCTURAL RESULTS}

In Section 3.2 expressions for the first few terms of the $\left\{A_{i}\right\}$ sequence were derived. By using those terms and working through the first few steps of the algorithm it can be shown, that, following simplification, for $i=1,2,3$,

$$
\begin{aligned}
& \left.{\underset{\sim}{\alpha}}_{i}^{\prime}=\left(\mu_{i 0} \stackrel{e}{\sim}^{\prime}+\mu_{i 1} \underset{\sim}{p}{ }_{1}^{\prime}+\ldots+\mu_{i i} \underset{\sim}{p}{ }_{i}^{\prime}\right) / \mu_{i i}\right) \\
& {\underset{\sim}{v}}_{i}^{\prime}=\left(\mu_{i 0} \stackrel{e}{\sim}^{\prime}+\mu_{i 1} \underset{\sim}{p}{ }_{1}^{\prime}+\ldots+\mu_{i i} \underset{\sim}{p} \underset{i}{\prime}\right) /\left(\mu_{i 0}+\mu_{i 1} p_{1, i+1}+\ldots+\mu_{i i} p_{i, i+1}\right) \text {, } \\
& {\underset{\sim}{\pi}}_{i}^{\prime}=\left(\mu_{i 0} e^{\prime}+\mu_{i 1} \underset{\sim}{p}{ }_{1}^{\prime}+\ldots+\mu_{i i} \underset{\sim}{p} \underset{i}{\prime}\right) /\left(m \mu_{i 0}+\mu_{i 1}+\ldots+\mu_{i i}\right),
\end{aligned}
$$

where $\mu_{10}=1-p_{11}$,

$$
\begin{aligned}
& \mu_{11}=1 \\
& \mu_{20}=\left(1-p_{11}\right)\left(1-p_{22}\right)-p_{12} p_{21}, \\
& \mu_{21}=1+p_{21}-p_{22}, \\
& \mu_{22}=1-p_{11}+p_{12},
\end{aligned}
$$




$$
\begin{aligned}
\mu_{30}= & \left(1-p_{11}\right)\left(1-p_{22}\right)\left(1-p_{33}\right)-p_{12} p_{23} p_{31}-p_{13} p_{21} p_{32} \\
& -p_{13}\left(1-p_{22}\right) p_{31}-\left(1-p_{11}\right) p_{23} p_{32}+p_{12} p_{21}\left(1-p_{33}\right) \\
\mu_{31}= & p_{21}\left(1-p_{33}+p_{32}\right)+\left(1-p_{22}\right)\left(1-p_{33}+p_{31}\right)+p_{23}\left(p_{31}-p_{32}\right) \\
\mu_{32}= & p_{31}\left(p_{12}-p_{13}\right)+p_{32}\left(1-p_{11}+p_{13}\right)+\left(1-p_{33}\right)\left(1-p_{11}+p_{12}\right) \\
\mu_{33}= & \left(1-p_{11}\right)\left(1-p_{22}+p_{23}\right)+p_{12}\left(p_{23}-p_{21}\right)+p_{13}\left(1-p_{22}+p_{21}\right) .
\end{aligned}
$$

The general structure exhibited by (5.1), and hence also by (5.2) and (5.3), holds for all $i=1,2, \ldots, m$. [A proof by induction shows that if (5.1) and (5.2) hold for $i=1, \ldots, n$ then, since

$$
\begin{aligned}
& \stackrel{p}{\sim}_{n+1}^{\prime} A_{n+1}={\underset{\sim}{p}}_{n+1}^{\prime}\left[I+B_{0}+\ldots+B_{n}\right] . \\
& =\underline{\sim}^{\prime}{ }_{n+1}\left[I+\underline{b}_{0}{\underset{v}{v}}_{0}^{\prime}+\ldots+\underset{\sim}{\sim} n \underset{\sim}{v}{ }_{n}^{\prime}\right] \text {, } \\
& =\underline{\sim}^{\prime}{ }_{n+1}+\left(\underline{\sim}^{\prime}{ }_{n+1} \underset{\sim}{\sim} 0\right){\underset{v}{v}}_{0}^{\prime}+\ldots+\left(\underline{\sim}^{\prime}{ }_{n+1} \stackrel{b}{\sim}_{n}\right){\underset{\sim}{v}}_{n}^{\prime},
\end{aligned}
$$

using (3.22) with $i=n,{\underset{\sim}{\alpha}}_{n+1}^{\prime}$ is a linear combination of ${\underset{\sim}{0}}_{0}^{\prime}, \ldots,{\underset{\sim}{v}}_{n}^{\prime}, \underline{\sim}^{\prime}{ }_{n+1}$, i.e. of $e^{\prime}, \underset{\sim}{p}{ }_{1}^{\prime}, \ldots,{\underset{\sim}{p}}^{\prime}{ }_{n+1}$. Furthermore, the coefficient of $\sim_{\sim}^{p}{ }_{n+1}$ is unity whereby establishing the general structure of $\alpha^{\prime} n+1$.]

Note also that, from (5.1) and (3.25), ${\underset{\sim}{\alpha}}_{i}^{\prime} \underset{\sim}{\stackrel{e}{\sim}}=1$, for $i=1, \ldots, m$ and thus

$$
\mu_{i 0}+\mu_{i 1} p_{1 i}+\ldots+\mu_{i i} p_{i i}=\mu_{i i}
$$

Further, for $i=1,2$ it can be shown, by direct verification, that

$$
\mu_{i 0}+\mu_{i 1} p_{i, i+1}+\ldots+\mu_{i i} p_{i, i+1}=\mu_{i+1, i+1}
$$

which implies that

$$
v_{i i}={\underset{\sim}{v}}_{i}^{\prime} \underset{i}{\sim}=\mu_{i i} / \mu_{i+1, i+1}
$$

and

$$
\alpha_{i, i+1}=\alpha_{i}^{\prime} \stackrel{e}{\sim}_{i+1}=\mu_{i+1, i+1} / \mu_{i i}
$$

results that it has not been possible to establish in general.

Let $(I-P)_{i}$ be the leading $i t h$ order principal submatrix of $I-P$ formed by deleting all but the first $i$ rows and columns then, for $i=1,2,3$,

$$
\mu_{i 0}=\operatorname{det}(I-P)_{i}
$$


For the special case when $m=3$, with the notation used earlier in this section, it can be verified that

$$
\begin{gathered}
\alpha_{i}^{\prime}=\left(\mu_{i 1}, \mu_{i 2}, \mu_{i 3}\right) / \mu_{i i}, \quad(i=1,2,3), \\
\stackrel{v}{\sim}_{i}^{\prime}=\left(\mu_{i 1}, \mu_{i 2}, \mu_{i 3}\right) / \mu_{i+1, i+1}, \quad(i=1,2), \\
\underline{\pi}_{i}^{\prime}=\left(\mu_{i 1}, \mu_{i 2}, \mu_{i 3}\right) /\left(\mu_{i 1}+\mu_{i 2}+\mu_{i 3}\right), \quad(i=1,2,3),
\end{gathered}
$$

where

$$
\begin{gathered}
\mu_{1}=1-p_{11}+p_{12}=\mu_{22}, \\
\mu_{13}=1-p_{11}+p_{13}, \\
\mu_{23}=\mu_{33}=3 \mu_{30} .
\end{gathered}
$$

Observe that $\underset{\sim}{\pi}{ }_{1}^{\prime}, \pi_{2}^{\prime}$ and $\underset{\sim}{\sim}{ }_{3}^{\prime}$ give, respectively, the stationary probability vectors of the Markov chains whose transition matrices are

$$
\left[\begin{array}{ccc}
p_{11} & p_{12} & p_{13} \\
1 / 3 & 1 / 3 & 1 / 3 \\
1 / 3 & 1 / 3 & 1 / 3
\end{array}\right], \quad\left[\begin{array}{ccc}
p_{11} & p_{12} & p_{13} \\
p_{21} & p_{22} & p_{23} \\
1 / 3 & 1 / 3 & 1 / 3
\end{array}\right], \quad \text { and } \quad\left[\begin{array}{ccc}
p_{11} & p_{12} & p_{13} \\
p_{21} & p_{22} & p_{23} \\
p_{31} & p_{32} & p_{33}
\end{array}\right]
$$

In examining (5.11), with $i=3$, it can be shown that $\mu_{3 j}=3 D_{j},(j=1,2,3)$, where $D_{j}$ is the determinant formed by striking out the $j$ th row and $j$ th column of $I-P$. This leads to an expression for the stationary probability vector of a general irreducible, three state, Markov chain as

$$
\pi^{\prime}=\left(D_{1}, D_{2}, D_{3}\right) / \sum_{j=1}^{3} D_{j}
$$

The natural extension of (5.12) for a general finite irreducible Markov chain is also true, such a result being attributed to Mihoc by Fréchet (1950) and rediscovered by Singer (1964).

Although the full details of a proof of the generalization of (5.12) using the techniques of this paper have not been worked out, it is conjectured that for an $m$-state chain $\mu_{m j}=m D_{j}$, (a result that holds for $m=2,3$ ), so that the procedures proposed in this paper appear to lead to an effective algorithmic construction of Mihoc's technique. 


\section{EXAMPLE}

As an illustration of the perturbation procedure, we compute the stationary probabilities for the irreducible five-state Markov chain example used in Sheskin (1985) which was taken from Kemeny and Snell $(1960)$ p. 199 . The $(1,1)$ entry has been changed to ensure that $P$ is in fact a stochastic matrix, so that $P$ is given by

$$
P=\left[\begin{array}{ccccc}
0.831 & 0.033 & 0.013 & 0.028 & 0.095 \\
0.046 & 0.788 & 0.016 & 0.038 & 0.112 \\
0.038 & 0.034 & 0.785 & 0.036 & 0.107 \\
0.054 & 0.045 & 0.017 & 0.728 & 0.156 \\
0.082 & 0.065 & 0.023 & 0.071 & 0.759
\end{array}\right] \text {. }
$$

The following calculations were performed on a MacIntosh computer using the Microsoft Excel software spreadsheet. Although the program yields expressions to 14 significant figures, the results have been rounded to 3 decimal places.

$$
\begin{aligned}
& \alpha_{1}^{\prime}=(1.000,0.202,0.182,0.197,0.264), \\
& \ddot{v}_{1}^{\prime}=(4.950,1.000,0.901,0.975,1.307) \text {, } \\
& {\underset{\sim}{2}}_{2}^{\prime}=(1.277,1.000,0.202,0.244,0.403), \\
& {\underset{v}{\sim}}_{2}^{\prime}=(6.309,4.939,1.000,1.203,1.991) \text {, } \\
& \sim_{3}^{\prime}=(1.593,1.232, \quad 1.000,0.302,0.571) \text {, } \\
& \ddot{v}_{3}^{\prime}=(5.275,4.079,3.311,1.000,1.890) \text {, } \\
& \approx_{4}^{\prime}=(1.717,1.295,0.935,1.000,0.705) \text {, } \\
& \ddot{v}_{4}^{\prime}=(2.434,1.836,1.326,1.418,1.000) \text {, } \\
& \alpha_{5}^{\prime}=(0.841,0.573,0.237,0.459,1.000) \text {. }
\end{aligned}
$$

From the above calculations, the stationary probabilities for each successive perturbed 
transition matrix can easily be calculated, yielding the following results:

$$
\begin{aligned}
& \pi_{1}^{\prime}=(0.542,0.109,0.099,0.107,0.143), \\
& \pi_{2}^{\prime}=(0.409,0.320,0.065,0.078,0.129) \text {, } \\
& \pi_{3}^{\prime}=(0.339,0.262,0.213,0.064,0.122) \text {, } \\
& \pi_{4}^{\prime}=(0.304,0.229,0.165,0.177,0.125) \text {, } \\
& \pi_{5}^{\prime}=(0.270,0.184,0.076,0.148,0.322) \text {. }
\end{aligned}
$$

\section{FINAL COMMENTS}

The initial choice of $P_{0}$ as $\underset{\sim}{\underset{\sim}{e}}{ }^{\prime} / m$ ensures that it is possible to start with an irreducible Markov chain whose stationary distribution is easily found without having to compute a matrix inverse or to solve a general set of linear equations. The fact that every element of $P_{0}$ is non-zero leads to a sequence of matrices $A_{1}, A_{2}, \ldots$ that are "dense". Is it possible to start with a different Markov chain, say one that is relatively sparse, whose stationary distribution is well known and such that, for the early recursions, the equivalent sequence $A_{1}, A_{2}, \ldots$ retains such a sparsity property?

The periodic Markov chain with entries $p_{i i+1}^{(0)}=1,(i=1,2, \ldots, m-1)$, and $p_{m 1}^{(0)}=1$ is a potential candidate for $P_{0}$, whose stationary probability vector is also $\pi_{0}^{\prime}=e^{\prime} / m$. Even if $\underset{\sim}{t} 0$ and $\underline{u}_{0}$ can be specified so that $A_{0}=\left[I-P_{0}+\underset{\sim}{\sim} 0{\underset{\sim}{u}}_{0}^{\prime}\right]^{-1}$ has a simple structure much care would be required in carrying out any sequential row modification with this $P_{0}$. For example if, for the specified $P$ transition matrix, $p_{12}=0$ then state 2 is never reached in the Markov chain with transition matrix $P_{1}$ violating the required irreducibility property of $P_{1}$.

The major advantage in choosing $P_{0}=\underset{\sim}{e} \underset{\sim}{\prime} / m$ is that the irreducibility of each $P_{i}$ transition matrix is guaranteed at each step of the procedure.

\section{REFERENCES}

[1] FRECHLET, M., Recherches Théoriques Modernes sur le Calcul des Probabilités, Vol. 2, 2nd ed., Gauthier-Villars, Paris, 1950.

[2] GOLUB, G.H., and VAN LOAN, C.F., Matrix Computations, The Johns Hopkins University Press, Bailtimore, 1983. 
[3] GRASSMAN, W.K., TAKSAR, M.I., and HEYMAN, D.P., Regenerative analysis and steady state distributions for Markov chains, Oper. Res. 39 (1985), pp. 1107-1116.

[4] HARROD, W.J. and PLEMMONS, R.J., Comparison of some direct methods for computing stationary distributions of Markov chains, SIAM J. Sci. Statist. Comput., 5 (1984), pp. 453-469.

[5] HUNTER, J.J., Generalized inverses and their application to applied probability problems, Linear Algebra Appl. 45 (1982), pp. 157-198.

[6] HUNTER, J.J., Stationary distributions of perturbed Markov chains, Linear Algebra Appl. 82 (1986), pp. 201-214.

[7] ISAACSON, E. and KELLER, H.B., Analysis of Numerical Methods, John Wiley and Sons, New York, 1966.

[8] KEMENY, J.G. and SNELL, J.L., Finite Markov Chains, Van Nostrand, Princeton, 1960.

[9] KOURY, J.R., MCALLISTER, D.F. and STEWART, W.J., Iterative methods for computing stationary distributions of nearly completely decomposable Markov chains, SIAM J. Alg. Disc. Meth., 5 (1984), pp. 164-186.

[10] MEYER, Jr., C.D., Uncoupling Markov chains and the Simon-Ando theory of nearly reducible systems, Centre for Research in Scientific Computation, North Carolina State Univ., Tech. Rep. 10018701, 1987.

[11] PAIGE, C.C., STYAN, G.P.H. and WACHTER, P.G., Computation of the stationary distribution of a Markov chain, J. of Statist. Comput. Simulation, 4 (1975), pp. 173-186.

[12] SHESKIN, T.J., A Markov chain partitioning algorithm for computing steady state probabilities, Oper. Res. 33 (1985), pp. 228-235.

[13] SINGER, A., The steady state probabilities of a Markov chain as a function of the transition probabilities, Oper. Res. 12 (1964), pp. 498-499. 